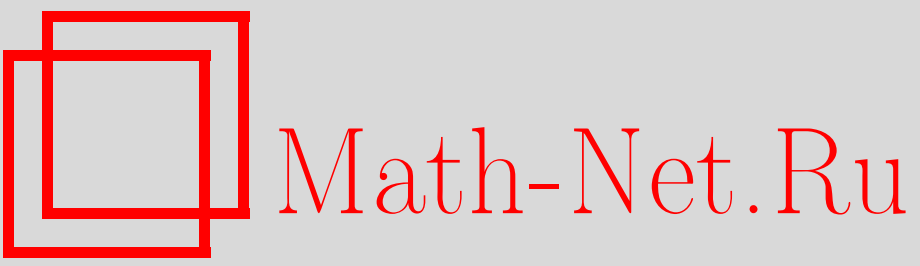

Т. Грава, Автомодельные асимптотические решения уравнений Уизема, УМН, 1999, том 54, выпуск 2, 169-170

DOI: https://doi.org/10.4213/rm138

Использование Общероссийского математического портала Math-Net.Ru подразумевает, что вы прочитали и согласны с пользовательским соглашением

http://www.mathnet.ru/rus/agreement

Параметры загрузки:

IP : 54.81 .137 .203

26 апреля 2023 г., 08:33:42 


\section{АВТОМОДЕЛЬНЫЕ АСИМПТОТИЧЕСКИЕ РЕШЕНИЯ УРАВНЕНИЙ УИЗЕМА}

\section{Т. ГРАВА}

Мы доказываем гипотезу С.П. Новикова, согласно которой решения уравнений Уизема с монотонными полиномиальными начальными данными имеют универсальную однофазную автомодельную асимптотику. Уравнения Уизема (см. [1]) представляют собой совокупность квазилинейных гиперболических систем вида

(1) $\frac{\partial u_{i}}{\partial t}+\lambda_{i}\left(u_{1}, u_{2}, \ldots, u_{2 g+1}\right) \frac{\partial u_{i}}{\partial x}=0, \quad x, t, u_{i} \in \mathbb{R}, \quad i=1, \ldots, 2 g+1, \quad g=0,1,2, \ldots$,

где $u_{1}>u_{2}>\cdots>u_{2 g+1}$. При данном $g$ система (1) называется $g$-фазными уравнениями Уизема. Нуль-фазные уравнения Уизема имеют вид $u_{t}+6 u u_{x}=0$ (где мы обозначили $u_{1}=u$ ). При $g>0$ скорости $\lambda_{i}\left(u_{1}, u_{2}, \ldots, u_{2 g+1}\right), i=1, \ldots, 2 g+1$, выражаются через полные гиперэллиптические интегралы рода $g$. Для монотонно убывающих начальных условий $x=\left.f(u)\right|_{t=0}$ решение нуль-фазного уравнения определяется уравнением характеристик $x=6 t u+f(u)$. Это решение глобально определено только при $0 \leqslant t<t_{c}$, где $t_{c}=\frac{1}{6} \min _{u \in \mathbb{R}}\left[-f^{\prime}(u)\right]-$ время градиентной катастрофы решения. При $t \geqslant t_{c}$ решение уравнений Уизема получается $C^{1}$-гладким склеиванием решений разных родов. Задача Коши для уравнений Уизема для монотонно убывающих гладких начальных данных $x=f(u)$ состоит в следующем.

При $t \geqslant 0$ плоскость $(x, t)$ разбивается на области $D_{g}$, где $g=0,1, \ldots$ В каждой области $D_{g}$ мы ищем решение $u_{1}(x, t)>u_{2}(x, t)>\cdots>u_{2 g+1}(x, t)$ для $g$-фазных уравнений Уизема (1). При каждом $t \geqslant 0$ функции $u_{1}(x, t)>u_{2}(x, t)>\cdots>u_{2 g+1}(x, t)$ можно изобразить на $(x, u)$-плоскости в виде ветвей многозначной функции. Решения уравнений Уизема с разными $g$ должны склеиваться так, чтобы получалась $C^{1}$-гладкая кривая в $(x, u)$-плоскости, гладко эволюционирующая по времени $t$. При $t=0$ имеется только область $D_{0}$ для всех $x$. Соответствующее нуль-фазное решение $u(x, t)$ нуль-фазного уравнения должно удовлетворять начальному условию $x=f(u(x, 0))$.

Мы скажем, что решение задачи Коши сушествует глобально и имеет род, не превосходящий $g_{0}$, если оно определено при всех $t>0$ и области $D_{g}$ пусты при $g>g_{0}$.

Список литературы по уравнениям Уизема можно найти в [2]. Задача Коши для уравнений Уизема была детально изучена Тяном в случае $g \leqslant 1$. Им были доказаны следующие теоремы.

Теорема 1 (см. [3]). Предположим, что монотонно убъвающие начальные данные $x=f(u)$ имеют лишь одну точку перегиба в начале координат на $(x, u)$-плоскости и удовлетворяют условию $f^{\prime \prime \prime}(u)<0$ при всех $u \neq 0$. Тогда решение уравнений Уизема существует при всех $t \geqslant 0$. Это решение имеет род один внутри интервала $x^{-}(t)<$ $x<x^{+}(t)$, где $x^{-}(t)<x^{+}(t)-$ две вещественнозначнье функции, удовлетворяющие условию $x_{-}(0)=x_{+}(0)=0$, и имеет род нуль вне әтого интервала.

Теорема 2 (см. [4]). Предположим, что решение задачи Коши для уравнений Уизема с монотонно убьвающими начальными данными $x=f(u)$ существует при любых $x, t \geqslant 0$. Пусть функция $f(u)$, определенная на всей вещественной оси, удовлетворяет условиям: $\lim _{u \rightarrow-\infty} f^{\prime \prime}(u)=+\infty ; f^{\prime \prime}(u)<0$ при $u \rightarrow+\infty ; f^{\prime \prime \prime}(u)<0$ при $u>u_{+}$, $u<u_{-}$, где $u_{+} \geqslant u_{-}$- некоторые вещественные числа. Тогда найдется время $T \geqslant 0$ такое, что при всех $t>T$ решение уравнений Уизема имеет род один внутри интервала $x^{-}(t)<x<x^{+}(t)$, где $x^{-}(t)<x^{+}(t)$ - две вещественнье функции от $t$, и имеет род нуль вне указанного интервала.

При $g=1$ скорости $\lambda_{i}\left(u_{1}, u_{2}, u_{3}\right), i=1,2,3$, в уравнениях Уизема (1) даются формулами [1]

$$
\lambda_{i}\left(u_{1}, u_{2}, u_{3}\right)=2\left(u_{1}+u_{2}+u_{3}\right)+4 \frac{\prod_{j \neq i}\left(u_{i}-u_{j}\right)}{u_{i}+\alpha_{0}}
$$

где $\alpha_{0}=\alpha_{0}\left(u_{1}, u_{2}, u_{3}\right)$ определяется полными эллиптическими интегралами. 
Для монотонно убывающих полиномиальных начальных данных степени $2 N+1$ вида $x=$ $f(u)=-u^{2 N+1}+c_{2 N} u^{2 N}+\cdots+c_{1} u+c_{0}$ решение одноффазного уравнения, которое непрерывно сшивается с нуль-фазным решением, имеет вид

$$
x=\lambda_{i} t+\left(\frac{1}{2} \lambda_{i}-u_{1}-u_{2}-u_{3}\right) \frac{\partial q}{\partial u_{i}}+q, \quad i=1,2,3 .
$$

Здесь $\lambda_{i}=\lambda_{i}\left(u_{1}, u_{2}, u_{3}\right)$ определяются формулами (3), а $q=\sum_{j=0}^{2 N+1} \frac{c_{k}}{Z_{k}} \Gamma_{k}$, где $Z_{k}=\frac{(2 k+1) ! !}{2^{k} k !}$ и $\Gamma_{k}=\Gamma_{k}\left(u_{1}, u_{2}, u_{3}\right)-$ коэффициенты разложения функции $\left(\left(\xi-u_{1}\right)\left(\xi-u_{2}\right)\left(\xi-u_{3}\right)\right)^{-1 / 2}=$ $\xi^{-3 / 2}\left(\Gamma_{0}+\Gamma_{1} \xi+\cdots+\Gamma_{k} \xi_{k}+\cdots\right)$ при $\xi \rightarrow \infty$.

Для начальных условий вида $f(u)=-u^{k}, k=3,5,7, \ldots$, получаются специальные автомодельные решения. Их род не превосходит единицы. Эти решения имеют вид $u_{i}(x, t)=$ $t^{\frac{1}{k-1}} U_{i}\left(t^{-\frac{k}{k-1}} x\right), i=1,2,3$. Действительно, при замене переменных $X=t^{-\frac{k}{k-1}} x, \quad u(x, t)=$ $t^{\frac{1}{k-1}} U\left(t^{-\frac{k}{k-1}} x\right), u_{i}(x, t)=t^{\frac{1}{k-1}} U_{i}\left(t^{-\frac{k}{k-1}} x\right), i=1,2,3$, система и уравнение характеристик $x=6 t u+f(u)$ превращаются в

(4) $X=\lambda_{i}(\vec{U})-\left[\frac{1}{2} \lambda_{i}(\vec{U})-U_{1}-U_{2}-U_{3}\right] \frac{\partial}{\partial U_{i}} \frac{\Gamma_{k}(\vec{U})}{Z_{k}}-\frac{\Gamma_{k}(\vec{U})}{Z_{k}}, \quad X=6 U-U^{k}$, где $\vec{U}=\left(U_{1}, U_{2}, U_{3}\right)$. Из теоремы 1 вытекает следующее утверждение.

СледСтвиЕ 1. Для $x=-u^{k}, k=3,5,7, \ldots$, уравнения Уизема имеют глобальное автомодельное однофазное решение $u_{1}>u_{2}>u_{3}, u_{i}(x, t)=t^{\frac{1}{k-1}} U_{i}\left(t^{-\frac{k}{k-1}} x\right), i=1,2,3$, внутри каспа на $(x, t)$-плоскости $x_{-}(k) t^{\frac{k}{k-1}}<x<x_{+}(k) t^{\frac{k}{k-1}}$, где $x_{-}(k)<x_{+}(k)-\partial в е$ вещественные константы, а $t>0$. На границе каспа однофазное решение сшивается $C^{1}$-гладким образом с решением $u(x, t)$ нуль-фазного уравнения. Константа $x_{-}(k)$ определяется из соотношения $x_{-}(k)=-6 \frac{k-1}{k}\left(2 z_{-}(k)-1\right)\left[\frac{6}{k}\left(1+2(k-1) z_{-}(k)\right)\right]^{\frac{1}{k-1}}$, $k=3,5,7, \ldots$, әде $z_{-}(k)>1$ - единственное вещественное решение уравнения $F\left(-k+2,2, \frac{5}{2} ; z\right)=0$. Здесь $F(a, b, c ; z)$ - гипергеометрическая функция. Константа $x_{+}(k)$ определяется из соотношения $x_{+}(k)=2 \frac{k-1}{k}\left(2 z_{+}(k)-3\right)\left[\frac{2}{k}\left(3+2(k-1) z_{+}(k)\right)\right]^{\frac{1}{k-1}}$, $k=3,5,7, \ldots$, где $z_{+}(k)>1$ - единственное вещественное решение уравнения $F\left(-k+2,2, \frac{7}{2} ; z\right)=0$.

ЗАмечАниЕ. При $k \rightarrow \infty$ решение (4) стремится к решению из [5] с начальными данными в виде ступеньки. Предельное решение теряет $C^{1}$-гладкость.

В соответствии с результатами [6], решение уравнений Уизема с полиномиальнтми начальными условиями существует при всех $x$ и при $t \geqslant 0$ и имеет род не больше $N$. Теорема 2 показывает, что существует время $T$ такое, что при всех $t>T$ решение уравнений Уизема с этими начальными условиями имеет род $g \leqslant 1$. Отсюда выводится следующая теорема.

Теорема 3. Решение уравнений Уизема (1) с полиномиальными начальными условиями при $t \rightarrow+\infty$ асимптотически стремится $\kappa$ автомодельному решению (4) $c$ начальными условиями $x=-u^{2 N+1}$.

\section{СПИСОК ЛИТЕРАТУРЫ}

[1] Whitham G. B. Linear and Nonlinear Waves. New York: Wiley, 1974. [2] Дубровин Б. А., Новиков С. П. // УМН. 1989. Т. 44. № 6. С. 29-98. [3] Tian F. R. // Comm. Pure Appl. Math. 1993. V. 46. P. 1093-1129. [4] Tian F. R. // Comm. Math. Phys. 1994. V. 166. P. 79-115. [5] Гуревич А. Г., Питаевский Л. П. // Письма ЖЭТФ. 1973. [6] Grava Т. // SISSA Preprint. 1997. 42/97/FM, solv-int/9707011.

Италия

E-mail: grava@sissa.it
Принято редколлегией 26.01.1999 